\title{
Simulation of the remanence influence on the transient states in a single-phase multiwinding transformer
}

\author{
Andrzej Wilk, Michal Michna \\ Gdansk University of Technology \\ Faculty of Electrical and Control Engineering \\ Narutowicza 11/12, 80-233 Gdańsk, Poland \\ e-mail:\{andrzej.wilk/michal.michna\}@pg.gda.pl
}

(Received: 22.09.2016, revised: 18.10.2016)

\begin{abstract}
This paper presents the mathematical model of a single-phase multi-winding core type transformer taking into account magnetic hysteresis phenomenon based on the feedback Preisach model (FPM). The set of loop differential equations was developed for a $K$-th winding transformer model where the flux linkages of each winding includes flux $\Phi$ common to all windings as a function of magneto motive force $\Theta$ of all windings. The first purpose of this paper is to implement a hysteresis nonlinearity involved in the $\Phi(\Theta)$ function which also accounts residual magnetic flux. The second purpose of this paper is experimental validation of the developed transformer model in a capacitor discharge test and several different values of residual magnetic flux.
\end{abstract}

Key words: single-phase transformer, magnetic flux common, magneto motive force, magnetic hysteresis, residual magnetic flux, capacitor discharge test

\section{Introduction}

One of many consequences of the magnetic hysteresis is the possibility of occurrence of residual magnetic flux density in the core of the transformer, whose value depends on several factors [1]: the magnetic properties of the core, the power factor at the time of shutdown of the transformer, the winding connections, etc. The existence of residual flux may be significant to the maximum value of the inrush current of the transformer and contribute to the undesirable effects of protection systems installed in the power system [2-4]. Effects of inrush currents are somewhat limited by the use of appropriate reactors and protection systems with time-lag characteristics. However, studies are carried out on the use of active control systems of the moment of a switching transformer, including the residual flux in the core $[2,5]$ to limit the inrush current. Accurate determination of the residual flux is a relatively difficult issue because it requires consideration of the hysteresis phenomenon and the eddy currents in the core. The accurate methods to prediction of the residual flux and inrush current of transformer 
circuits are therefore needed. It is expected that in this prediction method accurate mathematical circuit models of the transformers can be useful.

In this paper a single-phase multi-winding transformer with core type of construction is studied taking into account the hysteresis phenomenon of magnetic material and residual magnetic flux. Multi-winding transformers are used in central stations, power trains, and electronic devices. They enable to supply several circuits with different voltage and power level from a single source through a single physical device.

An arrangement of windings and iron core geometry of the considered transformer in cross section is shown in Fig. 1. This transformer has the tape wound core made by wrapping thin long grain oriented silicon steel strip around an appropriate path. The number of windings and arrangement of particular coils corresponds to a transformer used frequently in electric traction [6]. The coils denoted P2 and P4 are two sections of the primary traction winding. The coils denoted $\mathrm{P} 1$ and $\mathrm{P} 3$, connected in series inside the transformer, form a single section referred to as the primary auxiliary winding. The coils labeled T1 and T2, not connected inside the transformer, form two sections of the traction secondary windings. The coils S1 and S2, connected in series inside the transformer, are the auxiliary secondary winding.

Modeling of traction transformers is very important for simulations and analysis of transient states in power trains. The exact circuit parameters are needed for proper control strategy of drive systems [7] and for diagnosis purposes [8]. The key point of the transformer modeling is the representation of nonlinear magnetization and hysteresis of the iron core.

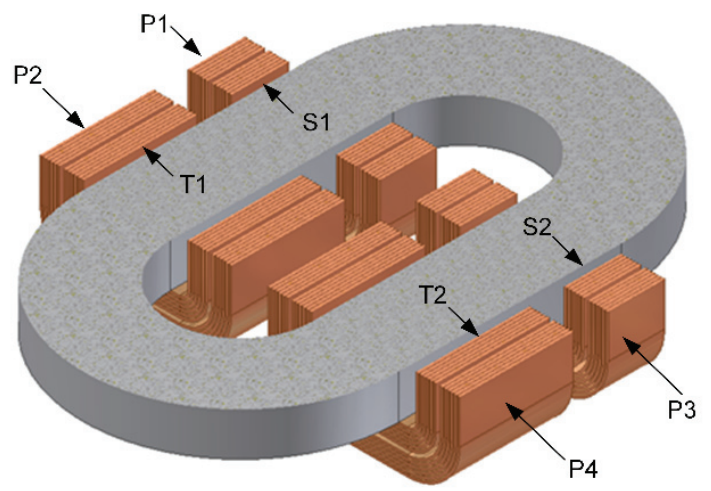

Fig. 1. Isometric view of cross section of the considered transformer and arrangement of its primary P1, P2, P3, P4 windings and secondary S1, S2, T1, T2 windings

\section{Macroscopic models of magnetic hysteresis}

Several efforts to model magnetic hysteresis have been reported until now. Among many models proposed so far, the hysteresis model based on Preisach's theory [9] and the JilesAtherton model [11] are proposed for accurate modeling and prediction of the magnetic characteristics. The Preisach model (PM) and Jiles-Atherton (J-A) model are classified into so-called macroscopic models of hysteresis [12]. 
In the J-A model the total magnetization in the magnetic material has two components reversible and irreversible. The J-A model can be used in analysis of coupled electromagnetic and thermal phenomena in the transformers [13]. It was reported in [14] that the J-M model has been successfully utilized to simulate of coupled electromagnetic, fluid dynamic and motion phenomena. This model can be used in FEM calculations [15] and recently, an algorithm and computer code was presented [16] to effectively determine several parameters of the J-A model.

In the PM the double integral of the Preisach distribution function $\mu(\alpha, \beta)$ is involved to find usually magnetic flux density $B$ as the function of the magnetic field intensity $H$. The induction $B$ depends not only on the magnetic field but also on the history of magnetization of the material. The PM had been initially utilized in the field of magnetism but its mathematical generality suggested implementation of this model in many areas of science. A pure mathematical form of the PM separated from its physical meaning was proposed by Krasnosel'skii [17]. This approach was further developed by Mayergoyz [18] for determining the conditions for the representations of the hysteresis nonlinearities and generalization of the PM.

Nowadays there are several generalizations of the original classical PM in other to improve its ability to represent complex experimental results. One possible solution to gain more accuracy for the representation of hysteresis nonlinearity is to use the so-called feedback Preisach model $[19,20]$. Description of most modifications of classical PM (generalized PM, moving PM, dynamic PM, vector PM) can be find in paper [21]. Many recent enhancements to the classical PM also allow simulation of coupled electromagnetic and thermal phenomena in magnetic materials. The PM has been used in this paper to simulate hysteresis effects in a single-phase transformer.

Experimental and simulation studies of the influence of the residual magnetic flux on the value of inrush current are rarely published and selected results are given in [3, 4, 22, 23]. In [3] and [4] lack a comparison of simulation results with experimental results, which do not allow to validate the models of hysteresis. In [22] the simplified model of the transformer was used, which does not take into account classical eddy current losses and anomalous losses. In [23] the components of classical and anomalous losses are included and show relatively good agreement between the results of simulation and experiment. However, in this hysteresis model, the shape of the small loops is deduced from the relevant geometric transformation of the rising and falling curve of the main hysteresis loop, which cannot be proven on the basis of the macroscopic model.

Two main objectives were formulated in this paper. The first objective concerned the development of a circuit model of the single-phase multi-winding transformer coupled with a generalized scalar Preisach hysteresis model. Equivalent circuit model of the transformer is developed in terms of the common magnetic flux $\Phi_{c}$ as function of ampere-turns $\Theta$ of all coils. In this paper hysteresis nonlinearity is involved in $\Phi_{c}(\Theta)$ expression. The new Preisach distribution function $\mu(\alpha, \beta)$ is proposed [24] as an analytical formula approximated by functional series. A hysteresis model with a feedback function as the third order polynomial is assumed in order to gain accuracy of the transformer model. The model also takes into account the equivalent circuit representing the classical and anomalous eddy current losses. 
The second objective concerned the simulations of the inrush current at different values of residual magnetic flux. The study of the transient state was based on the capacitor discharge through the primary windings at a predetermined value and sign of the residual magnetic flux in the core. This approach has provided a very good repeatability of the transient states, independent of the switch actuation time and physical phenomena on its contacts. Finally the simulation results predicted by the model have been successfully verified by experiments.

\section{Transformer equivalent circuit}

A schematic representation of the considered transformer with $K$-windings and their associated terminal voltages and the assumed current directions is shown in Fig. 2. For the transformer with $K=8$ windings operating in a power train, primary traction P1, P2, P3, and $\mathrm{P} 4$ coils (in parallel connection) are energized from a single AC voltage source. Secondary T1, T2, S1, and S2 coils are connected to power converters.

The set of loop differential equations of $K$-winding transformer model is expressed in matrix form as:

$$
\boldsymbol{u}=\boldsymbol{R} \boldsymbol{i}+\frac{\mathrm{d}}{\mathrm{d} t} \boldsymbol{\Psi}(\boldsymbol{i}),
$$

where: $\boldsymbol{u}=\left[u_{1}, u_{2}, \ldots, u_{K},\right]^{\mathrm{T}}$ is the vector of terminal voltages, $\boldsymbol{i}=\left[i_{1}, i_{2}, \ldots, i_{K},\right]^{\mathrm{T}}$ is the vector of winding currents and $\Psi(i)=\left[\Psi_{1}(i), \Psi_{2}(i), \ldots, \Psi_{K}(i)\right]^{\mathrm{T}}$ is the vector including the flux linkages in each winding.

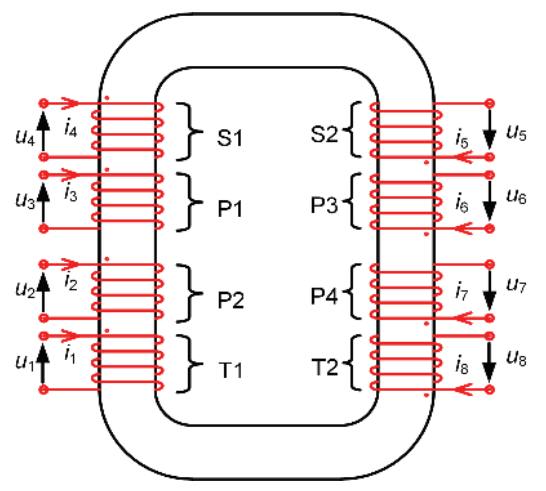

Fig. 2. Schematic representation of a single-phase and eight winding transformer: P1, P2, P3, and P4 - coils of primary winding (connected in parallel), $\mathrm{T} 1, \mathrm{~T} 2, \mathrm{~S} 1, \mathrm{~S} 2$ - secondary windings

The time rate of change of $\boldsymbol{\Psi}$ can be represented as:

$$
\frac{\mathrm{d}}{\mathrm{d} t} \boldsymbol{\Psi}(\boldsymbol{i}(t))=\left[\begin{array}{ccc}
\frac{\partial \Psi_{1}(\boldsymbol{i})}{\partial i_{1}} & \cdots & \frac{\partial \Psi_{1}(\boldsymbol{i})}{\partial i_{K}} \\
\vdots & & \vdots \\
\frac{\partial \Psi_{K}(\boldsymbol{i})}{\partial i_{1}} & \cdots & \frac{\partial \Psi_{K}(\boldsymbol{i})}{\partial i_{K}}
\end{array}\right] \frac{\mathrm{d}}{\mathrm{d} t}\left[\begin{array}{c}
i_{1} \\
\vdots \\
i_{K}
\end{array}\right]=\boldsymbol{L}_{\boldsymbol{d}}(\boldsymbol{i}) \frac{\mathrm{d}}{\mathrm{d} t} \boldsymbol{i},
$$


where $\boldsymbol{L}_{\boldsymbol{d}}(\boldsymbol{i})$ is the matrix of self and mutual dynamic inductances.

Recognizing that there is a flux common to all windings, the flux linkages of the $k$-th winding can be expressed by $[25,26]$ :

$$
\Psi_{k}(\boldsymbol{i})=N_{k} \Phi_{c}(\Theta(\boldsymbol{i}))+\sum_{n=1}^{K} L_{\sigma, k, n} i_{n}, \quad k, n=1,2, \ldots, K,
$$

where $\Phi_{c}$ is the flux common to all windings, $N_{k}$ is the number of turns of the $k$-th winding, $L_{\sigma, k, n}$ is the leakage inductance resulting from the existence of the leakage flux in the coil $k$ due to the flux generated by the current in the coil $n, \Theta$ is the total ampere turns of all windings defined by:

$$
\Theta(\boldsymbol{i})=N_{1} i_{1}+N_{2} i_{2}+, \ldots+N_{K} i_{K} .
$$

In this paper the relationship $\Phi_{c}(\Theta)$ accounts for nonlinear magnetic properties of the iron core and hysteresis effect.

\section{The feedback hysteresis model}

In the classical Preisach model a ferromagnetic material is made up of infinite set of magnetic dipoles (hysteresis operator), each having magnetic characteristics with two separate, randomly distributed properties $\alpha, \beta$ as shown in Fig. 3. Each operator has a rectangular hysteresis loop and is defined as mathematical operator $\gamma_{\alpha \beta}(H)$ that can assume only two values, +1 (positively switched) and -1 (negatively switched).

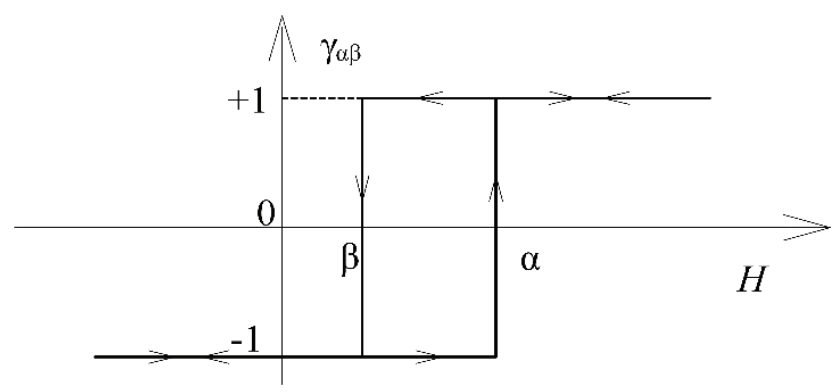

Fig. 3. Rectangular loop of elementary hysteresis operator

The relationship between magnetic field intensity $H$ and flux density $B$ in the classical Preisach model is expressed in the integral form as:

$$
B=\iint_{\alpha \geq \beta} \mu(\alpha, \beta) \gamma_{\alpha \beta}(H) \mathrm{d} \alpha \mathrm{d} \beta,
$$


where $\mu(\alpha, \beta)$ is a finite weight function having nonzero values within the limits of a major hysteresis loop. The term $\mu(\alpha, \beta)$ is also called the Preisach distribution function and can be regarded as a material constant. The weight function $\mu(\alpha, \beta)$ can be also treated as a probability density function where $\mu(\alpha, \beta) \operatorname{d} \alpha \mathrm{d} \beta$ equals the probability that a randomly selected operator has a rectangular loop $(\alpha, \beta)$. In the feedback Preisach model (FPM) the positive switching field $\alpha$ is replaced by $\alpha+H_{f}(B)$ and the negative switching field $\beta$ is replaced by $\beta+H_{f}(B)$ in Eq. (5).

In this paper the hysteresis nonlinearity is proposed to be involved in $\Phi_{c}(\Theta)$ expression. Hence, a block diagram representing the proposed hysteresis model taking into account $\Phi_{c}(\Theta)$ term is shown in Fig. 4.

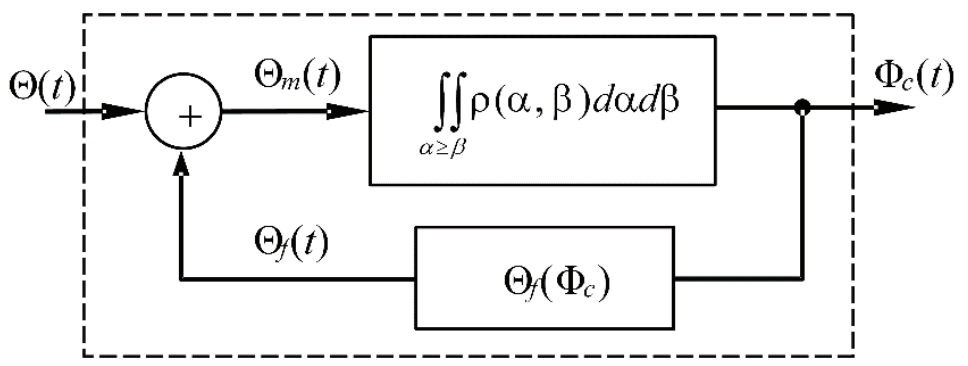

Fig. 4. Block diagram of the feedback hysteresis model

The upper rectangle shown in Fig. 4 represents the classical Preisach transducer [18]. The lower rectangle in feedback loop represents the contribution term $\Theta_{f}\left(\Phi_{c}\right)$ to the effective magnetomotive force $\Theta_{m}(t)=\Theta(t)+\Theta_{f}\left(\Phi_{c}\right)$ acting on elementary hysteresis operators. Hence, the scalar feedback Preisach model (FPM) is formulated by the following formula:

$$
\begin{aligned}
& \left.\Phi_{c}(t)=\iint_{\alpha \geq \beta} \rho\left[\alpha+\Theta_{f}(\Phi), \beta+\Theta_{f}(\Phi)\right)\right] \gamma_{\alpha, \beta}\left[\Theta_{m}(t)\right] \mathrm{d} \alpha \mathrm{d} \beta, \\
& \Theta_{m}=\Theta+\Theta_{f}\left(\Phi_{c}\right),
\end{aligned}
$$

where the $\rho\left(\alpha, \beta, \Theta\left(\Phi_{c}\right)\right)$ is the so called Preisach distribution function (PDF), which in this model also depends on the common flux $\Phi_{c}$ by a feedback relationship, $\gamma_{\alpha, \beta}(\Theta)$ describes the rectangular loop of elementary hysteresis operators.

Application of the FPM in a circuit simulation requires determination of $\rho\left(\alpha, \beta, \Theta\left(\Phi_{c}\right)\right)$ and $\Theta_{f}\left(\Phi_{c}\right)$ functions. An analytical approach for determination of feedback field contribution for $H_{f}(B)$ function is proposed in [27]. However this approach is applicable only for relatively small feedback factors. A complete parameter identification procedure of FPM is proposed in [28]. In this procedure however a linear feedback function is assumed and it may be applicable to some materials only. In [29] the nonlinear feedback function $H_{f}(B)$ is taken into account and the factorisation property of the function $\mu(\alpha, \beta)$ is assumed. However this is simplifying assumption and can be usable for a given class of magnetic materials.

In this paper a functional series of two-dimensional Gauss expressions to approximate the PDF is proposed [24] as: 


$$
\rho(\alpha, \beta)=\frac{1}{2 \pi} \sum_{n=1}^{N} \frac{A_{n}}{S_{x, n} S_{y, n}} \exp \left(\frac{-(\alpha+\beta)^{2}}{2 S_{x, n}^{2}}\right) \exp \left(\frac{-(\alpha-\beta)^{2}}{2 S_{y, n}^{2}}\right),
$$

where $A_{n}, S_{x, n}$, and $S_{y, n}$ are constant parameters.

A feedback function is proposed to be the third order polynomial:

$$
\theta_{f}\left(\Phi_{c}\right)=K_{1} \Phi_{c}+K_{3} \Phi_{c}^{3},
$$

where $K_{1}$ and $K_{3}$ are constant parameters.

\section{Parameter identification of the feedback Preisach model}

Identification of the FPM requires determination of $A_{n}, S_{x, n}, S_{y, n}, K_{1}$, and $K_{3}$ parameters by means of a convenient set of measured data. Measurements were carried out on a single-phase and eight-winding transformer (Fig. 5). The apparent power of P2, P4, T1, and T2 coils is $6.4 \mathrm{kVA}$, with a rated voltage of $230 \mathrm{~V}$. The apparent power of P1, P3, S1, and S2 coils is $3.2 \mathrm{kVA}$, with a rated voltage of $115 \mathrm{~V}$. Rated currents of all coils are the same $-28 \mathrm{~A}$. The iron core of the tested transformer is made with a grain oriented silicon steel strip of ET11427 type. Thickness of the strip is equal to $0.27 \mathrm{~mm}$.

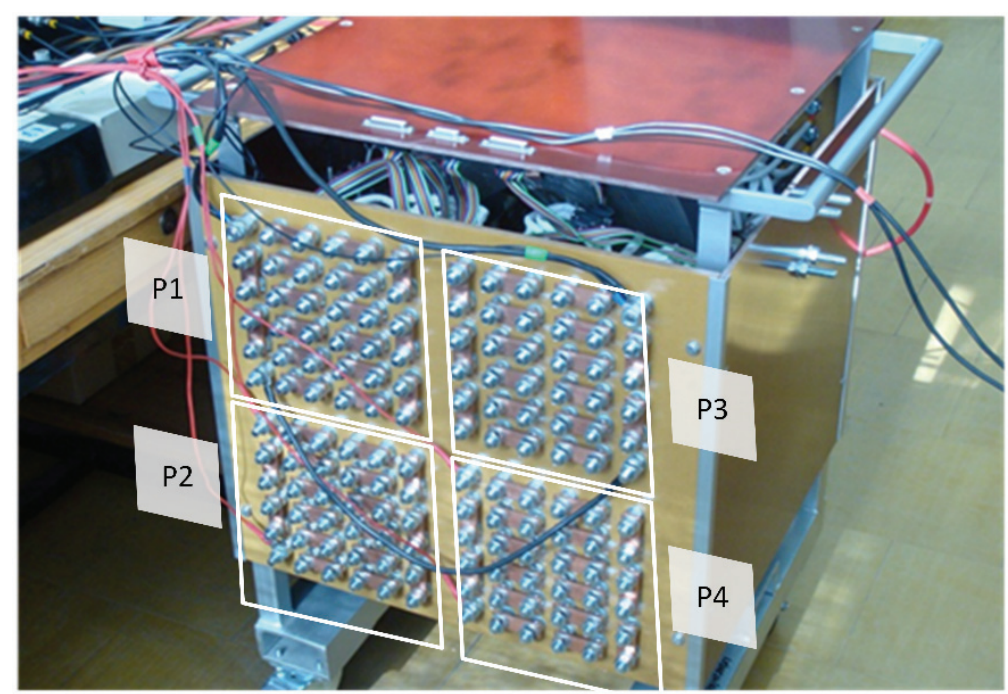

Fig. 5. Single-phase tested transformer with eight windings as part of experimental setup for measurements of hysteresis loops

The magnetizing windings were energized by laboratory power supply of PS 8000 DT type (Elektro-Automatik $\mathrm{GmbH}$ ) controlled by a function generator of AFG3011 type (Tektronix, Inc.). The hysteresis $\Phi_{c}(\Theta)$ characteristics of the tested transformer are calculated from the set 
of measured voltages and currents [30,31]. The flux density is obtained by numerical integration of the voltage induced in the measurement coil. The core was demagnetized before measurements. Measurements have been done under slow time varying excitation current. The frequency equal to $0.020 \mathrm{~Hz}$ for the major loop was applied in order to reduce the dynamic effects in magnetic material.

For determinations of $A_{n}, S_{x, n}, S_{y, n}, K_{1}$, and $K_{3}$ parameters the Levenberg-Marquardt optimization algorithm [32] was used. Only initial and limiting descending $\Phi_{c}(\Theta)$ curves were utilized for calculation of these parameters. Parameter values of the FPM obtained from the identification procedure are shown in Table 1.

Table 1. Parameter values of the FPM - unit of $S_{x}$, and $S_{y}$ is ampere

\begin{tabular}{l|l|l|l|l|l|l|l|l}
\hline $\boldsymbol{S}_{x, \mathbf{1}}$ & $\boldsymbol{S}_{x, \mathbf{2}}$ & $\boldsymbol{S}_{x, \mathbf{3}}$ & $\boldsymbol{S}_{\boldsymbol{v}, \mathbf{1}}$ & $\boldsymbol{S}_{v, \mathbf{2}}$ & $\boldsymbol{S}_{v, \mathbf{3}}$ & $\boldsymbol{A}_{\mathbf{1}}$ & $\boldsymbol{A}_{\mathbf{2}}$ & $\boldsymbol{A}_{\mathbf{3}}$ \\
\hline 14.3 & 99.8 & 1770 & 29.3 & 34.5 & 16.74 & 0.024 & 0.013 & 0.030 \\
\hline
\end{tabular}

The value of $K_{1}=720$ and $K_{3}=-25.9 \mathrm{E}+06$. A surface plot of the PDF of the tested transformer core is shown in Fig. 6.

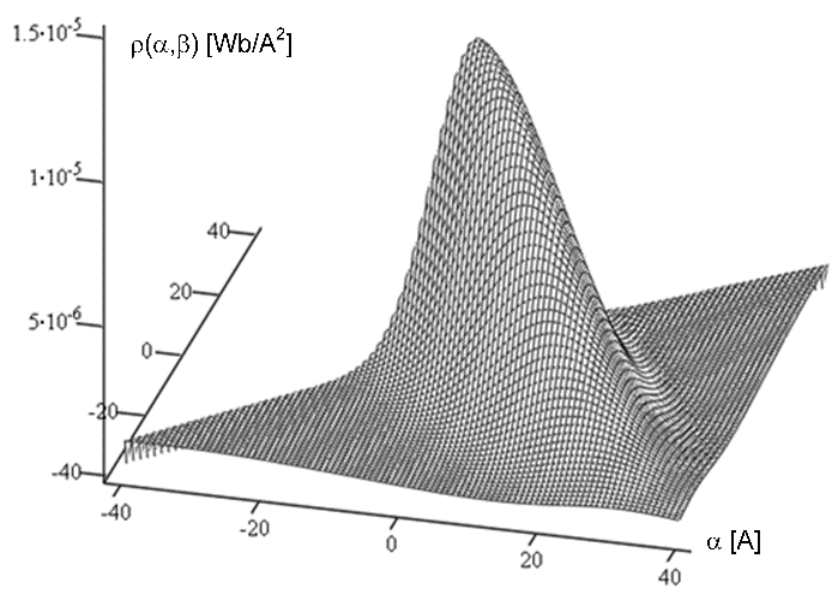

Fig. 6. Surface plot of the PDF of the tested transformer core

\section{Experimental and simulation results of magnetic hysteresis}

As an example, the FPM has been applied for representation of some symmetrical major loops at different total ampere-turns $\Theta$. Fig. 7 shows the simulated symmetrical major loop compared with the experimental one at the range of ampere turns from 0 to $2600 \mathrm{~A}$. In Fig. 8 the same simulated and measured downward and upward trajectories are shown at the range of ampere turns from 0 to $250 \mathrm{~A}$. Differences between measured and simulated hysteresis loops are relatively small. 


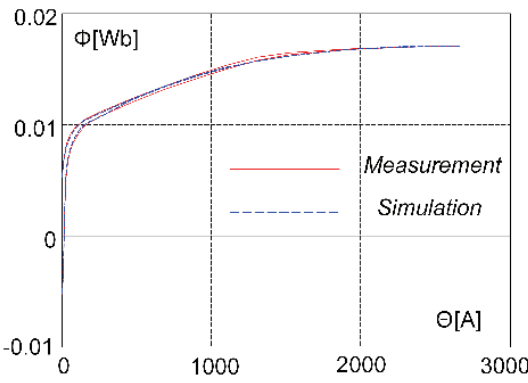

Fig. 7. Simulated and measured trajectories of symmetrical major hysteresis loops - verification of applied model

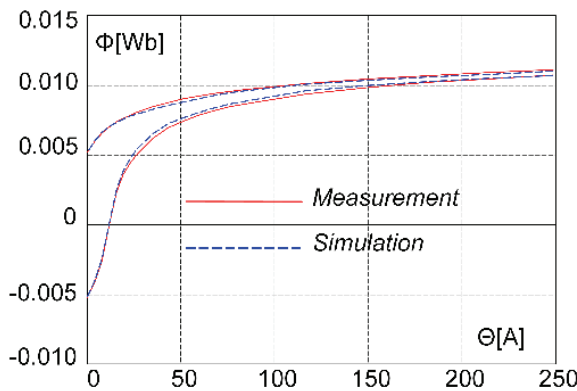

Fig. 8. Simulated and measured parts of symmetrical major hysteresis loops - verification of applied model at the range of ampere turns from 0 to $250 \mathrm{~A}$

\section{Circuit simulations of transformer coupled with the feedback hysteresis model}

In this chapter, simulations taking into account the hysteresis model are performed for the tested transformer, described in chapter 4 (Fig. 5). In order to demonstrate the hysteresis effect in inrush current the capacitor discharge test at no-load transformer operation is assumed. An equivalent circuit of the transformer at considered test is shown in Fig. 9. The schematic arrangement of core and primary windings for this test is shown in Fig. 1.

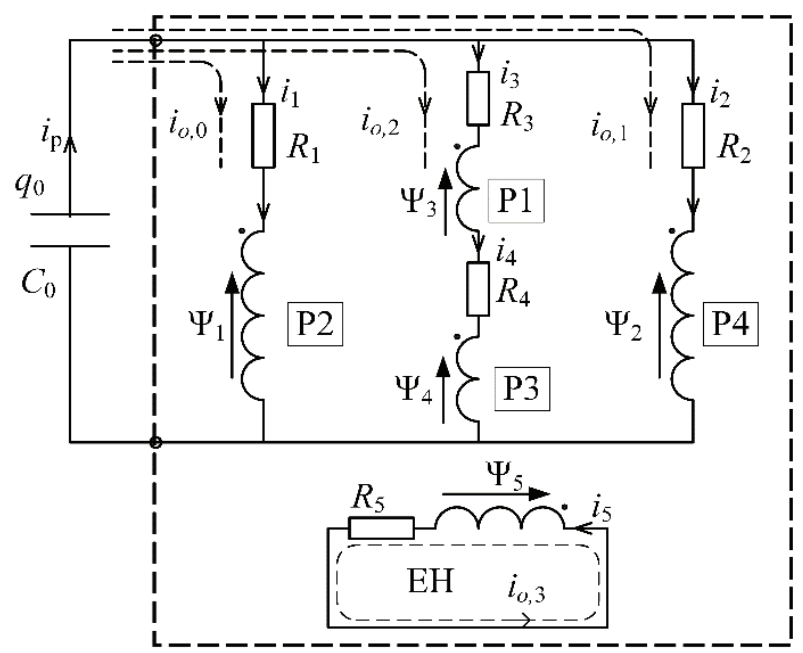

Fig. 9. Equivalent circuit of the considered transformer supplied from voltage source at no load operation

The primary windings $(\mathrm{P} 1+\mathrm{P} 3, \mathrm{P} 2, \mathrm{P} 4)$ are energized from the capacitor having capacitance $C_{0}$ with the initial charge $q_{0}$. A single equivalent circuit $\mathrm{EH}$ for modeling eddy current losses and an additional loss component (excess loss) is assumed. The rest of particular sym- 
bols are as follows: $\Psi_{1}$ is the flux linkage of P2 winding; $\Psi_{2}$ is the flux linkage of P4 winding; $\Psi_{3}$ is the flux linkage of P1 winding; $\Psi_{4}$ is the flux linkage of P3 winning; $\Psi_{5}$ is the flux linkage of the equivalent loop representing eddy currents and excess losses; $R_{1}$ is the resistance of $\mathrm{P} 2$ winding; $R_{2}$ is the resistance of $\mathrm{P} 4$ winding; $R_{3}$ is the resistance of $\mathrm{P} 1$ winding; $R_{4}$ is the resistance of $\mathrm{P} 3$ winding; $R_{5}$ is the resistance of equivalent loop representing eddy currents and excess losses.

Mathematical description of the equivalent circuit shown in Fig. 9 that takes into account all magnetic couplings between the transformer windings has the following general form:

$$
\left(\boldsymbol{C}_{u}^{\boldsymbol{T}} \boldsymbol{L}_{\boldsymbol{d}}\left(\boldsymbol{i}_{\boldsymbol{o}}\right) \boldsymbol{C}_{u}\right) \frac{\mathrm{d}}{\mathrm{d} t} \boldsymbol{i}_{\boldsymbol{o}}+\left(\boldsymbol{C}_{u}^{\boldsymbol{T}} \boldsymbol{R} \boldsymbol{C}_{u}\right) \boldsymbol{i}_{\boldsymbol{o}}=\frac{-q_{0}}{C_{0}}\left[\begin{array}{llll}
1 & 1 & 1 & 0
\end{array}\right]^{\boldsymbol{T}},
$$

where the matrix of dynamic inductances $\boldsymbol{L}_{\boldsymbol{d}}$, the matrix of resistances $\boldsymbol{R}$, and the matrix of constraints $\boldsymbol{C}_{\boldsymbol{u}}$ are defined as follows:

$$
\boldsymbol{L}_{\boldsymbol{d}}=\left[\begin{array}{lllll}
\frac{\partial \Psi_{1}}{\partial i_{1}} & \frac{\partial \Psi_{1}}{\partial i_{2}} & \frac{\partial \Psi_{1}}{\partial i_{3}} & \frac{\partial \Psi_{1}}{\partial i_{4}} & \frac{\partial \Psi_{1}}{\partial i_{5}} \\
\frac{\partial \Psi_{2}}{\partial i_{1}} & \frac{\partial \Psi_{2}}{\partial i_{2}} & \frac{\partial \Psi_{2}}{\partial i_{3}} & \frac{\partial \Psi_{2}}{\partial i_{4}} & \frac{\partial \Psi_{2}}{\partial i_{5}} \\
\frac{\partial \Psi_{3}}{\partial i_{2}} & \frac{\partial \Psi_{3}}{\partial i_{3}} & \frac{\partial \Psi_{3}}{\partial i_{4}} & \frac{\partial \Psi_{3}}{\partial i_{5}} \\
\frac{\partial \Psi_{4}}{\partial i_{1}} & \frac{\partial \Psi_{4}}{\partial i_{2}} & \frac{\partial \Psi_{4}}{\partial i_{3}} & \frac{\partial \Psi_{4}}{\partial i_{4}} & \frac{\partial \Psi_{4}}{\partial i_{5}} \\
\frac{\partial \Psi_{5}}{\partial i_{1}} & \frac{\partial \Psi_{5}}{\partial i_{2}} & \frac{\partial \Psi_{5}}{\partial i_{3}} & \frac{\partial \Psi_{5}}{\partial i_{4}} & \frac{\partial \Psi_{5}}{\partial i_{5}}
\end{array}\right], \quad \boldsymbol{R}=\left[\begin{array}{ccccc}
R_{1} & 0 & 0 & 0 & 0 \\
0 & R_{2} & 0 & 0 & 0 \\
0 & 0 & R_{3} & 0 & 0 \\
0 & 0 & 0 & R_{4} & 0 \\
0 & 0 & 0 & 0 & R_{5}
\end{array}\right], \boldsymbol{C}_{\boldsymbol{u}}=\left[\begin{array}{llll}
1 & 0 & 0 & 0 \\
0 & 1 & 0 & 0 \\
0 & 0 & 1 & 0 \\
0 & 0 & 1 & 0 \\
0 & 0 & 0 & 1
\end{array}\right]
$$

The matrix of constraints establishes a relation between the so called non-generalized coordinates (set of currents $-i_{1}, i_{2}, i_{3}, i_{4}, i_{5}$ ) and generalized coordinates (set of loop currents $\left.-i_{o, 1}, i_{o, 2}, i_{o, 3}, i_{o, 4}\right)$ included in vector $\boldsymbol{i}_{\boldsymbol{o}}$. The relation between these coordinates is given by:

$$
\left[\begin{array}{lllll}
i_{1} & i_{2} & i_{3} & i_{4} & i_{5}
\end{array}\right]^{T}=\boldsymbol{C}_{\boldsymbol{u}}\left[\begin{array}{llll}
i_{o, 0} & i_{o, 1} & i_{o, 2} & i_{o, 3}
\end{array}\right]^{T} .
$$

Assuming that there is a flux common to all $\mathrm{P} 2, \mathrm{P} 4, \mathrm{P} 1$, and $\mathrm{P} 3$ windings, the matrix of dynamic inductances is expressed by:

$$
\boldsymbol{L}_{\boldsymbol{d}}=\frac{\partial \Phi_{c}}{\partial \Theta}\left[\begin{array}{lllll}
N_{1} N_{1} & N_{1} N_{2} & N_{1} N_{3} & N_{1} N_{4} & N_{1} N_{5} \\
N_{2} N_{1} & N_{2} N_{2} & N_{2} N_{3} & N_{2} N_{4} & N_{2} N_{5} \\
N_{3} N_{1} & N_{3} N_{2} & N_{3} N_{3} & N_{3} N_{4} & N_{3} N_{5} \\
N_{4} N_{1} & N_{4} N_{2} & N_{4} N_{3} & N_{4} N_{4} & N_{4} N_{5} \\
N_{5} N_{1} & N_{5} N_{2} & N_{5} N_{3} & N_{5} N_{4} & N_{5} N_{5}
\end{array}\right]+\left[\begin{array}{lllll}
L_{\sigma, 1,1} & L_{\sigma, 1,2} & L_{\sigma, 1,3} & L_{\sigma, 1,4} & L_{\sigma, 1,5} \\
L_{\sigma, 2,1} & L_{\sigma, 2,2} & L_{\sigma, 2,3} & L_{\sigma, 2,4} & L_{\sigma, 2,5} \\
L_{\sigma, 3,1} & L_{\sigma, 3,2} & L_{\sigma, 3,3} & L_{\sigma, 3,4} & L_{\sigma, 3,5} \\
L_{\sigma, 4,1} & L_{\sigma, 4,2} & L_{\sigma, 4,3} & L_{\sigma, 4,4} & L_{\sigma, 4,5} \\
L_{\sigma, 5,1} & L_{\sigma, 5,2} & L_{\sigma, 5,3} & L_{\sigma, 5,4} & L_{\sigma, 5,5}
\end{array}\right],
$$

where $N_{1}=190$ is the number of turns of P2 winding, $N_{2}=190$ is the number of turns of P4 winding, $N_{3}=95$ is the number of turns of $\mathrm{P} 1$ winding, and $N_{4}=95$ is the number of turns of $\mathrm{P} 3$ winding. 
The matrix of leakage inductances of the considered transformer was defined by Wilk [26] for which the numerical values given in Henrys are:

$$
\left[\begin{array}{lllll}
L_{\sigma, 1,1} & L_{\sigma, 1,2} & L_{\sigma, 1,3} & L_{\sigma, 1,4} & L_{\sigma, 1,5} \\
L_{\sigma, 2,1} & L_{\sigma, 2,2} & L_{\sigma, 2,3} & L_{\sigma, 2,4} & L_{\sigma, 2,5} \\
L_{\sigma, 3,1} & L_{\sigma, 3,2} & L_{\sigma, 3,3} & L_{\sigma, 3,4} & L_{\sigma, 3,5} \\
L_{\sigma, 4,1} & L_{\sigma, 4,2} & L_{\sigma, 4,3} & L_{\sigma, 4,4} & L_{\sigma, 4,5} \\
L_{\sigma, 5,1} & L_{\sigma, 5,2} & L_{\sigma, 5,3} & L_{\sigma, 5,4} & L_{\sigma, 5,5}
\end{array}\right]=10^{-3}\left[\begin{array}{ccccc}
13.7 & 1.23 & 3.05 & 0.1 & 0 \\
1.23 & 13.7 & 0.1 & 3.05 & 0 \\
3.05 & 0.1 & 4.26 & 0.4 & 0 \\
0.1 & 3.05 & 0.4 & 4.26 & 0 \\
0 & 0 & 0 & 0 & 0
\end{array}\right] .
$$

The resistances of the considered transformer are as follows (given in Ohms): $R_{1}=0.347$; $R_{2}=0.347 ; R_{3}=0.201 ; R_{4}=0.201 ; R_{5}=5490$. The procedure for the determination of the value of $R_{5}$ was reported in [33].

In this model the relationship $\Phi_{c}(\Theta)$ that accounts for nonlinear magnetic properties of the iron core and hysteresis effect given by Eqs. (6), (7), and (8) is implemented.

\section{Measurements and simulation results}

Experimental studies consisted of the discharge of the capacitor through the primary winding at several different values of the residual magnetic flux. The use of a capacitor as a power source has provided a relatively high repeatability of the measured transient states independent of the moment of switching and the phenomena at the terminals of the connector [33]. The measurement results of inrush currents are shown in Fig. 10 and the simulation results of inrush currents are shown in Fig. 11. All measurements and simulations were performed at the capacitor $C_{0}$ charged to a voltage of $200 \mathrm{~V}$.

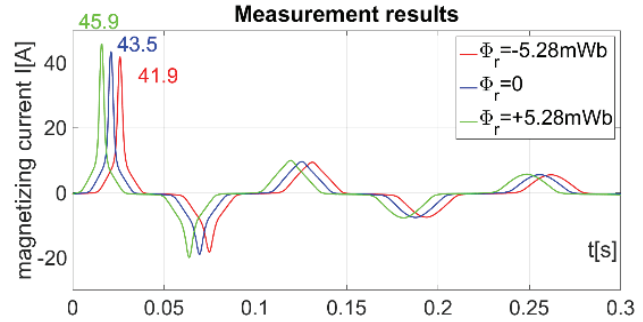

Fig. 10. The results of measurements of the transient state of the magnetizing current $i_{p}$, at the different values of residual magnetic flux

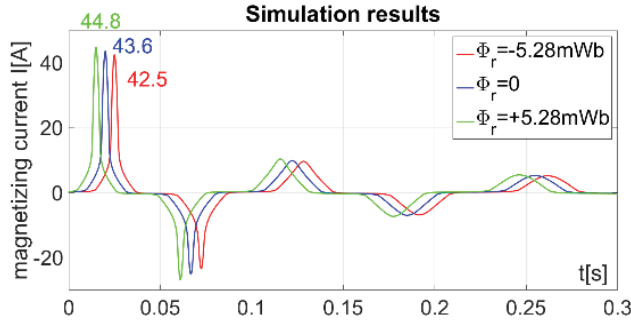

Fig. 11. The results of simulation of the transient state of the magnetizing current $i_{p}$, at the different values of residual magnetic flux

The results are given for three different residual magnetic flux values $\Phi_{r}=5.28 \mathrm{mWb}$, $\Phi_{r}=0 \mathrm{mWb}$, and $\Phi_{r}=-5.28 \mathrm{mWb}$. A positive value of $\Phi_{r}$ means that the initial magnetic field direction excited by current $i_{p}$ is consistent with the direction of residual magnetic flux density 
$B_{r}$. In the case of negative values of $\Phi_{r}$, the initial direction of the magnetic field excited by current $i_{p}$ is opposite.

The differences between measurements and simulations are relatively small. The differences between the waveforms of the exciting currents can be reduced by using nonlinear resistance $R_{5}$ in the equivalent loop.

\section{Conclusions}

In this paper the ability of the scalar feedback Preisach model to predict hysteretic behaviour between the common flux $\Phi_{c}$ and the total ampere turns $\Theta$ in the singe-phase and multiwinding transformer was implemented. The numerical implementation of the applied model is based on the new analytical distribution function $\rho(\alpha, \beta)$ defined as the functional series of two-dimensional Gauss expressions. The feedback function is represented by the third order polynomial. Hysteresis model is coupled with circuit model of the transformer.

Simulation of the free oscillation waveform in a capacitor discharge test is a relatively critical test of the model. The amplitude of the subsequent oscillations are properly modelled and correspond to the value obtained in the measurements. Good agreement between the phase relationship of simulation and measurement results is also important. This proves the correct approach of classical and anomalous losses in the transformer model. The simulation results indicate the usefulness of the applied circuit modeling methodology for the simulation of the transformer systems in a transient state, taking into account magnetic hysteresis and remanence.

\section{References}

[1] Ling P.C.Y., Basak A., Investigation of magnetizing inrush current in a single-phase transformer, IEEE Transactions on Magnetics, vol. 24, no. 6, pp. 3217-3222 (1988).

[2] Brunke J.H., Frölich K.J., Eliminations of transformer inrush currents by controlled switching Part II: Application and performance considerations, IEEE Transactions on Power Delivery, vol. 16, no. 2, pp. 281-285 (2001).

[3] Lin C.E., Cheng C.L., Huang C.L., Yeh J.C., Investigation of magnetizing inrush current in transformers. Part I - Numerical simulation, IEEE Transactions on Power Delivery, vol. 8, no. 1, pp. 246-253 (1993).

[4] Teape J.W., Slater R.D., Simpson R.R., Wood W.S., Hysteresis effects in transformers, including ferroresonance, Proceedings of IEE, vol. 123, no. 2, pp. 153-158 (1976).

[5] Abdulsalam S.G., Xu W., A sequential phase energization method for transformer inrush current reduction - Transient performance and practical considerations, IEEE Transactions on Power Delivery, vol. 22, no. 1, pp. 208-216 (2007).

[6] Hayek J.E., Transformer design as a key for efficiency optimization, XIX Inter. Conf. on Electrical Machines (ICEM-2010), Rome, Italy, pp. 1-4 (2010).

[7] Jager W.A.G., and Tubbing G.H., A vector oriented control strategy for a 4-quadrant line side converter, Fifth European Conference on Power Electronics and Applications (1993), Brighton, pp. 213-218 (1993).

[8] Wilk A., Internal winding fault detection in a traction transformer using a real-time reference model, Electromotion, vol. 17, no. 1, pp. 37-46 (2010). 
[9] Preisach F., Über die magnetische Nachwirkung, Zeitschrift für Physik, Bd.94, 1935, pp. 274-302 (1935).

[11] Jiles D.C., Atherton D.L., Ferromagnetic hysteresis, IEEE Transactions on Magnetics, vol. 19 no. 5, pp. 2183-2185 (1983).

[12] Liorzu F., Phelps B., Atherton D.L., Macroscopic models of magnetization, IEEE Transactions On Magnetics, vol. 36, no. 2, pp. 418-428 (2000).

[13] Łyskawiński W., Sujka P., Szeląg W., Barański M., Numerical analysis of hysteresis loss in pulse transformer, Archives of Electrical Engineering, vol. 60, no. 2, pp. 187-195 (2011)

[14] Jędryczka C., Sujka P., Szelag W., The influence of magnetic hysteresis on magnetorheological fluid clutch operation, COMPEL: The International Journal for Computation and Mathematics in Electrical and Electronic Engineering, vol. 26 no. 2, pp. 711-721 (2009).

[15] Gyselinck J., Dular P., Sadowski N., Leite J., Bastos J., Incorporation of a Jiles-Atherton vector hysteresis model in 2D FE magnetic field computations, COMPEL: The International Journal for Computation and Mathematics in Electrical and Electronic Engineering, vol. 23 no. 3, pp. 685-93 (2004).

[16] Knypiński Ł., Nowak L., Sujka P., Radziuk K., Application of a PSO algorithm for identification of the parameters of Jiles-Atherton hysteresis model, Archives of Electrical Engineering, vol. 61, no 2, pp. 139-148 (2012).

[17] Krasnosel'skii M.A., Pokrovskii A.V., Sistemy s gisterezisom (Systems with Hysteresis), Nauka, Moskow (1983).

[18] Mayergoyz I.D., Mathematical models of hysteresis, IEEE Transactions on Magnetics, vol. MAG-22, no. 5, pp. 603-608 (1986).

[19] Brokate M., Della Tore E., The wiping-out property of the moving model, IEEE Transactions on Magnetics, vol. 27 no. 5, pp. 3811-3814 (1991).

[20] Kadar G., Della Torre E., Hysteresis modeling I: Noncongruency, IEEE Transactions on Magnetics, vol. 23, no. 5, pp. 2820-2822 (1987).

[21] Iványi A., Füzi J., Szabó Z., Preisach models of ferromagnetic hysteresis, Electrical Review (Poland), R. LXXIX 3/2003, pp. 145-150 (2003).

[22] Adly A.A., Hanafy H.H., Abu-Shady S.E., Utilizing Preisach models of hysteresis in the computation of three-phase transformer inrush currents, Electric Power System Research, vol. 65, pp. 233-238 (2003).

[23] Faiz J., Saffari S., Inrush current modeling in a single-phase transformer, IEEE Transactions on Magnetics, vol. 46, no. 2, pp. 578-581 (2010).

[24] Wilk A., Representation of magnetic hysteresis in a circuit model of a single-phase transformer, COMPEL: The International Journal for Computation and Mathematics in Electrical and Electronic Engineering, vol. 34, no. 3, pp. 778-791 (2015).

[25] Wilk A., Nieznanski J., Moson I., Nonlinear model of a wound iron core traction transformer with the account of magnetic hysteresis, XIX International Conference on Electrical Machines (ICEM2010), Rome, Italy, pp. 1-7 (2010).

[26] Wilk A., Pokonski R., Determination of leakage inductances of multi-winding and single-phase transformer, The Scientific Papers of Faculty And Control Engineering, Gdansk University of Technology, no. 31, Gdansk, Poland 2011, pp. 145-150 (2011).

[27] Mayergoyz I.D., Adly A.A., Numerical Implementation of the feedback Preisach model, IEEE Transactions on Magnetics, vol. 28, no. 5, pp. 2605-2607 (1992).

[28] Della Torre E., Vajda F., Parameter identification of the complete-moving-hysteresis model using major loop data, IEEE Transactions on Magnetics, vol. 30 no. 6, pp. 3811-3814 (1994).

[29] Ragusa C., An analytical method for the identification of the Preisach distribution function, Journal of Magnetism and Magnetic Materials, vol. 254-255, pp. 259-261 (2003).

[30] Dolinar M., Dolinar D., Štumberger G., Polajžer B., Ritonja J., A three-phase core-type transformer iron core model with included magnetic cross saturation, IEEE Transactions on Magnetics, vol. 42, no. 10, pp. 2849-2851 (2006). 
[31] Fuchs E.F., You Y., Measurement of $\lambda$-i characteristics of asymmetric three-phase transformers and their applications, Proc. Ninth International conference on Harmonics and Quality of Power, Orlando, Florida, USA, pp. 91-96 (2000).

[32] More J.J., The Levenberg-Marquardt algorithm: Implementation and theory. Lecture Notes in Mathematics, Numerical Analysis, vol. 630, pp. 105-116 (1978).

[33] Wilk A., Michna M., Cichowski A., Simulation of the remanence influence on the transient states of the single-phase transformer including feedback Preisach model, 40th Annual Conference of the IEEE Industrial Electronics Society (IECON-2014), Dallas, TX, USA, pp. 875-880 (2014). 